\title{
Le rire des féministes
}

\author{
The laughter of feminists
}

\section{Laurie Laufer}

Centre de Recherches Psychanalyse Médecine et Société, UFR Etudes psychanalytiques, Université Paris Diderot, Francia aurie.laufer@univ-paris-diderot.fr

\section{RÉSUMÉ:}

En France, dans le sillage du mouvement de Mai 68, émerge spontanément et joyeusement le Mouvement de Libération des Femmes (MLF), à l'initiative d'Antoinette Fouque et de Monique Wittig. Au cœur de leur débat, de leur relecture critique des textes freudiens et lacaniens se trouvent pensés à nouveau frais le discours et la théorie de la psychanalyse sur "La femme", "le féminin", "la sexualité féminine", "la différence des sexes". Les dogmes théoriques concernant "le continent noir" romantiquement évoqués ainsi par Freud sont revisités, voire renversés. Les querelles autour du terme même de "féminisme" produisent et ouvrent à d'autres discours. Freud a toujours considéré que la théorie psychanalytique était open to revision et Lacan que la psychanalyse gagnerait à être en "extension". Que se passe-t-il lorsque la théorie et la pratique psychanalytiques rencontrent les discours féministes? L'horizon s'ouvre-t-il pour le discours psychanalytique, sa théorie, sa pratique? Une ouverture, une révision peuventelle s'opérer? Un renversement du dogme, une nouvelle joie de la pratique analytique?

Mots ClÉs: Psychanalyse, Féminisme, Différentialisme, Matérialisme, Sexualité, Genre.

\section{ABstraCT:}

In France, in the wake of the movement of May 68, the Women's Liberation Movement (MLF) spontaneously and joyfully emerged, initiated by Antoinette Fouque and Monique Wittig. In their debates and their critical rereadings of the Freudian and Lacanian texts, they think afresh the discourse and theory of psychoanalysis on "Women", "the feminine", "female sexuality", and "sex difference". The theoretical dogmas concerning "the black continent" romantically evoked by Freud are revisited, and even reversed. The quarrels over the very term of "feminism" produce and give way to other discourses. Freud always considered that psychoanalytical theory was open to revision and Lacan that psychoanalysis would benefit from being in 'extension'. What happens when psychoanalytic theory and practice meet feminist discourses? Does this encounter imply that the horizon should open up for psychoanalytical discourse, its theory and practice? Can an opening, a revision then take place? A dogma, a new joy in analytical practice?

KeYwords: Psychoanalysis, Feminism, Differentialism, Materialism, Sexuality, Gender.

\section{INTRODUCTION}

"J'écris de chez les moches, pour les moches, les vieilles, les camionneuses, les frigides, les mal baisées, les imbaisables, les hystériques, les tarées, toutes les exclues du grand marché à la bonne meuf. (...)

Parce que l'idéal de la femme blanche, séduisante mais pas pute, bien mariée mais pas effacée, travaillant sans trop réussir pour ne pas écraser son homme, mince mais pas névrosée par la nourriture, restant indéfiniment jeune sans se faire défigurer par les

chirurgiens de l'esthétique, maman épanouie mais pas accaparée par les couches et les devoirs d'école, bonne maîtresse de maison mais pas bonniche traditionnelle, cultivée mais moins qu'un homme, cette femme blanche heureuse qu'on nous brandit tout le temps sous le nez, celle à laquelle on devrait faire l'effort de ressembler, à part qu'elle a l'air de

beaucoup s'emmerder pour pas grand-chose, de toutes façons je ne l'ai jamais croisée, nulle part. Je crois bien qu'elle n'existe pas".

(Virginie Despentes, King-Kong Théorie, 2006, p. 9-11 et 14) 
Deux séquences freudiennes révèlent des articulations possibles entre psychanalyse et féminisme(s). La première est un épisode qui figure dans Les études sur l'hystérie écrites par Freud en 1895. Ce moment est d'une grande importance dans le processus d'invention de la psychanalyse, non seulement parce qu'il éclaire ce que va être la méthode analytique mais aussi parce qu'il représente une véritable rupture avec ce qui se pratiquait à l'époque de Charcot et de ses patientes hystériques.

\section{EMmY ET Sidonie, LES PARLEUSES ${ }^{1}$}

Freud est en séance avec Emmy Von N. qui souffre, entre autres symptômes, de douleurs gastriques qui l'empêchent de dormir. Dans Études sur l'hystérie, il écrit:

"Par un détour quelconque, j'arrivai à lui demander comment ses douleurs gastriques étaient survenues et d'où elles provenaient [...]. Avec assez de réticences, elle me répond qu'elle n'en sait rien. Je lui donne jusqu'à demain pour s'en souvenir. Elle me dit alors d'un ton très bourru, qu'il ne faut pas lui demander toujours d'où provient ceci ou cela mais la laisser raconter ce qu'elle a à dire. J’y consens et elle poursuit sans préambule" (Freud, 1990 [1995], p. 48. C'est moi qui souligne).

Ce consentement est un renversement des positions entre le médecin sachant et savant et la patiente. Emmy Von N. a des choses à dire, et elle dit qu'elle a des choses à dire et à faire entendre. Elle parle. "Il se trouve que les femmes parlent, elles aussi", dit Lacan. "On peut le regretter, mais c'est un fait. Elle veut donc, elle aussi, l'objet, et même un objet, en tant qu'elle ne l'a pas. On nous en rebat assez les oreilles avec l'histoire du Penisneid"2 (Lacan, 2004 [1962-1963], p. 233). Lacan fait ce constat dans le contexte d'un séminaire consacré notamment au contre- transfert "féminin" et à son érotisation chez l'analyste Lucy Towe. ${ }^{3}$ Les femmes parlent elles aussi, consent-il, et pas simplement juste par "envie du pénis".

L'autre séquence pouvant mettre en perspective les prémisses d'une réflexion féministe de ou sur la psychanalyse est la suivante: Freud reçoit en séance une jeune femme de 18 ans qu'il est convenu désormais d'appeler "la jeune homosexuelle".

"L'analyse se déroula pour ainsi dire sans le moindre indice de résistance. L'analysée était très coopérante du point de vue intellectuel, mais sans se départir de sa tranquillité d'âme. Un jour que je lui expliquais un point de théorie particulièrement important et qui la concernait de près, elle me fit cette répartie sur un ton inimitable: "Ah, mais c'est très intéressant !" telle une dame du monde que l'on promène dans un musée et qui considère avec son face-à-main des objets qui lui sont parfaitement indifférents" (Freud, 1992 [1920], p. 262).

Très manifestement, la jeune femme se moque des prétentions au savoir de Freud. Selon la théorie que lui présente celui-ci, elle serait déçue que sa mère attende un enfant de son père, alors que c'est elle qui souhaitait cet enfant. Oui très intéressant, dit-elle, jolie théorie, se moque-t-elle... Sidonie Csillag aime la baronne Puttkamer, et elle est prête à être conciliante avec son père : elle cède à sa demande et accepte d'aller voir Freud. Mais Sidonie n'est pas comme Dora : le père œedipien ne l'intéresse pas. ${ }^{4}$ Dans leur livre consacré à Sidonie Csillag, Ines Rieder et Diana Voigt (2003) relatent un autre épisode. Après une séance chez Freud, la baronne Puttkamer demande à Sidonie:

“Alors raconte ma chère $e^{5}[. .$.$] Tu sais qu'il m'interroge depuis quelque temps sur mes parents et sur mes frères, et qu'il veut$ tout savoir sur eux. C'est sur le plus jeune qu'il s'est surtout acharné la dernière fois. Imagine ce qu'il m'a dit aujourd'hui : que j'aurais aimé avoir un enfant de mon père, et évidemment comme c'est ma mère qui l'a eu, je la hais à cause de ça et mon père aussi, c'est pour ça que je me détourne complètement des hommes... C'est révoltant!” (Rieder et Voigt, 2003, p. 66).

Ines Rieder et Diana Voigt ont pu rencontrer Gretl (Sidonie). Lors de leur entretien, dont l'enregistrement est inédit, ${ }^{6}$ Sidonie fait cette réflexion: 


\begin{abstract}
"Quand je réfléchis, Freud ne pouvait pas être complètement idiot. Et comme il n'était pas idiot, il a dû savoir et reconnaître que dans l'innocence de mes dix-neuf ans j'étais comme une enfant de cinq ans. Je le suis resté longtemps. Complètement innocente. Est-ce que l'on dit de telles choses à une enfant totalement innocente? Que peut-être dans mon subconscient j'aurais souhaité avoir avec mon père l'enfant que ma mère a eu quand j'avais dix-sept ans. Un homme ne peut pas inventer quelque chose de plus ordurier. Et il a eu le culot de dire ça à une jeune fille de dix-neuf ans dont il devait savoir qu'elle était complètement innocente" (Entretien avec Ines Rieder et Diana Voigt, 1997, enregistrement non publié).7
\end{abstract}

La figure de la jeune homosexuelle a fait l'objet d'un commentaire dans le séminaire de Lacan, La relation d'objet:

"Il s'agit d'expliquer [pour Freud], en quoi la situation a été sans issue à l'intérieur du traitement, et il nous le dit. C'est pour autant que la résistance n'a pas été vaincue, que tout ce qu'on a pu lui dire n'a jamais fait que l'intéresser énormément, sans qu'elle abandonne ses positions dernières, à savoir qu'elle a maintenu tout cela, comme on dirait aujourd'hui, sur le plan d'un intérêt intellectuel. Il compare la personne dans ses réactions à peu près à la dame à qui on montre des objets divers, et qui à travers son face-à-main dit : 'Comme c'est joli!'” (Lacan, 1994 [1957], p. 106).

Dans ces deux séquences ici rapprochées, parole et savoir se trouvent contestés ou réappropriés par Emmy et Sidonie. De cela, Freud en convient. Certains commentaires issus de la théorie psychanalytique ont pu voir là la confirmation de l'hystérie : Emmy et Sidonie défieraient la parole du "père", elles seraient les bravaches critiques, les provocatrices viriles, les pétroleuses ironistes, prêtes à contredire le "Maître" pour mieux le faire choir. C'est à cela, dit encore la doxa analytique, qu'on reconnaît les hystériques, dans une analyse circulaire, sans extériorité. Fermez le ban, circulez, il n'y a plus rien à comprendre, CQFD.

Pourtant, Freud comme Lacan peuvent parfois entendre les marges et les biais de leur théorie en mouvement. Ainsi, Freud (1985 [1926]) a toujours considéré que la théorie psychanalytique était "open to revision" (p. 17). Pour Lacan, la psychanalyse gagnerait à être en "extension”:

"Qu'y renonce donc plutôt, dit-il, celui qui ne peut rejoindre à son horizon la subjectivité de son époque. Car comment pourrait-il faire de son être l'axe de tant de vies, celui qui ne saurait rien de la dialectique qui l'engage avec ces vies dans un mouvement symbolique?" (Lacan, 1966 [1953], p. 321).

Que peut-il alors se passer lorsque la théorie et la pratique psychanalytiques rencontrent les discours féministes? L'horizon s'ouvre-t-il pour le discours psychanalytique, sa théorie, sa pratique? Une ouverture, une révision peuvent-elles s'opérer?

\title{
3. Tout geste est RenVersement (Wittig, 1969, P. 7)
}

Un détour sur les premières occurrences du mot féministe semble ici nécessaire. Le terme de "féministe" a émergé en France d'un double mouvement quasiment simultané: on le trouve en 1871 dans une thèse de médecine soutenue par Valère Faneau de la Cour intitulée Du Féminisme et de l'infantilisme chez les tuberculeux, ce qui ferait de son auteur l'inventeur du mot "féminisme" (Fraisse, 2007; Bard et Chaperon, 2017). Dans ce contexte, le terme a un sens médical et biologique: il désigne la présence de caractères "féminins" chez un homme en raison d'une maladie tuberculeuse qui provoque un "arrêt de développement" du corps (d'où l'association avec "l'infantilisme") et une "masculinisation incomplète".

Mais, à la même époque, le terme connaît une diffusion plus vaste sous la plume d'Alexandre Dumas fils. En 1872, il publie un pamphlet, intitulé L'homme-femme, Réponse à M. Henri d'Ideville, dans le contexte d'une affaire de mœurs et d'un débat sur l'adultère et l'interdiction du divorce. Ce débat tire son origine d'un fait divers, l'assassinat d'une épouse adultère par son mari. Cet événement nommé l'affaire Dubourg suscita de vives réactions et d'importantes polémiques. L'enjeu est celui de positions morales par rapport à la liberté sexuelle de la femme. Dans un article paru dans le journal Le Soir du 6 juillet 1872, Henri d'Ideville prône le pardon de la femme adultère et Dumas-fils lui réplique par L'Homme-Femme. À la première question qu'il pose "Faut-il tuer la femme adultère ? Faut-il lui pardonner?", Dumas-fils (1872) répond sans équivoque: 
“Si j'avais un fils, je lui dirais : ce n'est pas la femme, ce n'est même pas une femme ; elle n'est pas dans la conception divine, elle est purement animale [...] tue-la” (p. 176). Voilà le ton de l'affaire.

“Les féministes, passez-moi ce néologisme, disent, à très bonne intention d'ailleurs : tout le mal vient de ce qu'on ne veut reconnaître que la femme est l'égale de l'homme et qu'il faut lui donner la même éducation et les mêmes droits qu'à l'homme ; l'homme abuse de sa force, etc., etc. Nous nous permettrons de répondre aux féministes que ce qu'ils disent là n'a aucun sens. La femme n'est pas une valeur égale, supérieure ou inférieure à l'homme, elle est une valeur d'un autre genre, comme elle est un être d'une autre forme et d'une autre fonction. La preuve qu'elle n'est pas aussi forte que l'homme, c'est qu'elle se plaint toujours de ce que l'homme est plus fort qu'elle; or si la nature a donné la force à l'homme, c'est pour qu'il s'en serve, comme il doit se servir de tous les dons qu'il a reçus pour l'œuvre qu'il a à faire. En effet, un des premiers usages que le masculin a fait de sa force a été d'enfermer et de subordonner le plus possible le féminin, dont il a besoin dans certains cas, s'étant aperçu qu'il lui en coûte cher, à lui masculin, quand ce féminin est en liberté, même dans un paradis” (Dumas-fils, 1872, p. 91-93).

Pour le promoteur du terme "féministe", les hommes ainsi qualifiés sont "des superficiels et des tendres" (Dumas-fils, 1872, p. 99) - des termes plus près de l'injure que de la louange, en ce temps, et l'implicite ne fait pas de doute. Alexandre Dumas-fils utilise ce terme pour dénigrer et stigmatiser les hommes qui souhaitent l'émancipation des femmes, ces hommes "féministes" sont des efféminés.

Si le terme "féministe" fut inventé par deux hommes - l'un dans une perspective médicale et nosographique, l'autre dans une perspective moraliste et dépréciative envers des hommes - c'est à Hubertine Auclert que revient l'usage du terme dans son sens politique actuel (Hause, 2007). Hubertine Auclert, que l'histoire retient comme "la suffragette française", s'empare de l'insulte et la retourne: elle se présente, selon ses propres termes, comme une "féministe intégrale".

La condition de production du terme "féministe" annonce son histoire et peut-être sa postérité. D'insulte et/ou de qualification pathologique, il devient politique dans un geste de renversement, de détournement : la reprise réitérée le resignifie et produit la subversion de son sens initial (Butler, 2005 [1990]). Ainsi, selon l'histoire du mot, une position féministe est celle du détournement, du retournement, de la resignification. Dans le contexte contemporain, la notion d'empowerment ("capacitation", "développement du pouvoir d'agir", "autonomisation", "responsabilisation", ou "émancipation”) signifie ce retournement, cette réappropriation subjective. La notion d'empowerment a émergé aux États- Unis dans des situations de luttes pour les droits civiques et de combats féministes et LGBTQ+. La réappropriation des termes stigmatisant a été une stratégie de lutte, une agency, une capacité à faire d'une insulte un blason, d'une honte une fierté. Queer, pédé, gouine sont autant de termes dont la réappropriation par les personnes concernées participe d'un geste de subjectivation. L'historienne Sylvie Chaperon (2017) relate un moment de ces luttes, en France, où un tel renversement de l'insulte se produit : Monique Wittig, sa sœur Gilles, ainsi que deux féministes radicales familières du women's lib américain, Margaret Stephenson et Marcia Rosthenburg, organisent un coup d'éclat, "une première sortie publique à l'université de Vincennes le 21 mai 1970 arborant des tee-shirts provocants: 'nous sommes toutes des mal baisées, des hystériques, des prostituées" (p. 596).

Peu de temps auparavant, en 1969, Monique Wittig avait publié Les Guérillères, un ouvrage titré d'un néologisme inventé par l'auteure: mal baisées, hystériques, prostituées, gouines rouges sont en marche, ainsi qu'elle l'écrit sous la forme poétique et en lettres majuscules comme incipit de cet ouvrage:

\author{
CONNIVENCES RÉVOLUTIONS \\ C'EST L'ARDEUR AU COMBAT \\ CHALEUR INTENSE MORT ET BONHEUR \\ DANS LES POITRINES MAMELÉES \\ LES PHÉNIX LES PHÉNIX LES PHÉNIX \\ CÉLIBATAIRES ET DORÉS LIBRES \\ ON ENTEND LEURS AILES DÉPLOYÉES \\ LES OISEAUX LES SIRÈNES NAGEANTES \\ LES ARÊTES TRANSLUCIDES LES AILES \\ LES SOLEILS VERTS LES SOLEILS VERTS \\ LES PRAIRIES VIOLETTES ET PLATES
}




\section{LES CRIS LES RIRES LES MOUVEMENTS \\ ELLES AFFIRMENT TRIOMPHANT \\ QUE TOUT GESTE EST RENVERSEMENT.}

Les femmes "guérillères" partent au combat "avec ardeur" dans "les cris et les rires" et d'un mouvement spontané qui émerge, elles font naître des cendres du phénix des femmes aux corps triomphants.

C'est ainsi que le Mouvement de Libération des femmes (MLF) émerge spontanément en France en octobre 1968, dans les traces de Mai et de la réactivation de la lutte des classes et des luttes anti-impérialistes dont il est issu. "À l'initiative d'Antoinette Fouque, de Monique Wittig et de Josiane Chanel, une quinzaine de femmes se réunissent à Paris dans un studio de la rue Vaugirard prêté par Marguerite Duras” (MLF, 2018a, p. 17).

Le discours et la théorie de la psychanalyse sur "La femme", "le féminin", "la sexualité féminine" sont au cœur de leurs débats, tout comme les dogmes théoriques concernant "le continent noir", tel que l'évoque romantiquement Freud. Juliet Mitchell fait le constat suivant, dès 1974:

"Pour la grande majorité du mouvement féministe, Freud fut et demeure l'ennemi. On admet généralement que pour la psychanalyse les femmes sont des êtres inférieurs qui ne peuvent parvenir à la véritable féminité qu'en tant qu'épouses et que mères. On y voit également une justification du statu quo patriarcal et bourgeois, et on fait du personnage même de Freud l'incarnation de toutes ces infamies. Certes ceci est vrai d'une certaine vulgarisation des théories de Freud" (p. 13).

En France, Simone de Beauvoir considérait déjà, dès 1949, qu'il était difficile de discuter 'la psychanalyse': "Comme toutes les religions - christianisme, marxisme - elle se montre, écrit-elle sur un fond de concepts rigides, d'une souplesse gênante » (p. 81). Ainsi, par exemple, lorsque la psychanalyste Karen Horney, exilée aux États-Unis, critique la notion d' "envie du pénis » et qu'elle prend ses distances à l'égard de l'orthodoxie freudienne, elle est exclue du New York Psychoanalytic Institute en 1941.

\section{In OU OUT : PSYCHANALYSE OU FÉMINISME}

Dans les années 1970, stimulées par l'émergence festive et joyeuse, spontanée et désordonné du MLF, les femmes, dans des réunions non mixtes, prennent les textes, les lisent et les critiquent : Marx, Engels, Freud, et d'autres sont retournés, renversés, sans censure. l'instar des trois mots qui clôturent une conférence de Foucault sur "l'herméneutique de soi" prévaut la méthode du "refus, (de) la curiosité et (de) l'innovation". Les femmes ne se laissent plus seulement être l'objet d'un discours: c'est une pensée critique qui se met en place.

Mais si Antoinette Fouque et Monique Wittig ont contribué collectivement à l'émergence du MLF, des divergences théoriques apparaissent. La sexualité, l'homosexualité, les moyens de lutte pour la libération des femmes et la psychanalyse sont l'objet de tensions. Contre toute attente, la déchirure se consomme à partir du mot féminisme lui-même. Cette fracture dessine la frontière entre ce que l'on a appelé au sein du MLF le courant différentialiste Psychanalyse et politique, Psychépo, créé par Antoinette Fouque et le courant du féminisme matérialiste, représenté par Monique Wittig.

Le courant différentialiste Psychanalyse et politique rejette le terme même de féminisme. Il tente d'articuler la théorie psychanalytique de l'inconscient en tant qu'analyse de discours normatifs à la question politique de la différence des sexes. Psychanalyse et politique a été, dans les années 1970, en France, une tentative de reconsidérer la théorie et la pratique psychanalytique dans leur versant subversif en les délestant de leurs scories œdipianisantes et familialistes et de leurs apories patriarcales. Influencé par le "retour à Freud" prôné par Jacques Lacan, PsychéPo tente de redonner à la notion de "féminin" la spécificité, la prévalence, voire les avantages que la psychanalyse semblait effacer. Il s'agit de revaloriser la "géni(t)alité de la femme", selon l'expression de Antoinette Fouque, par la maternité, par la puissance procréatrice de "la" femme.

Cette pensée de la différence des sexes - dont un certain nombre de psychanalystes féministes se réclament, telles Luce Irigaray, Michèle Montrelay, Sarah Koffman, Julia Kristeva, ainsi que l'écrivaine proche de la 
psychanalyse, Hélène Cixous - critique et souhaite déconstruire l'idée freudienne d'une libido dont la nature serait masculine. ${ }^{8}$ Dans les années 1980, dans divers dits et écrits, Antoinette Fouque propose ces formules: "libido 2 femelle, utérine, originelle" ou "libido creandi", préservant et maintenant le discours théorique psychanalytique d'une psychogénèse et d'une psychosexualité. Il y a deux sexes, il y a deux libidos, telle est la théorie d'Antoinette Fouque (2004 [1995]).

Dans un mouvement symétriquement inverse de celui de Freud, Antoinette Fouque propose, dans sa lecture de “l'envie du pénis", de considérer “l'envie d'utérus chez les hommes” (2004 [1995]) perpétuant ainsi l'idée d'une différence des sexes marquée par le manque biologique ou organique chez chacun des sexes. Avec PsychéPo, elle tente d'ériger $L a$ différence des sexes comme programme politique : oui, dit-elle en substance, la femme est différente de l'homme en ce qu'elle, et elle seule, peut porter un enfant. Dans cette perspective, cette puissance procréatrice est la cause de la volonté de domination par les hommes impuissants à enfanter. Cette "génitalité", que Antoinette Fouque n’hésite pas à faire glisser du côté du géni(t)al, est l'affirmation de l'être-femme. Cette pensée critique se déploie à l'intérieur du corpus psychanalytique qu'il s'agit de réviser, d'élargir, voire de renverser: "Je voyais, écrit Antoinette Fouque, que la psychanalyse était aussi une religion phallocentrique ou une pensée de domination, quoi qu'on dise" (MLF, 2018a, p. 34).

Dans ce contexte, il s'agit de faire surgir "le sujet-femme" de ces analyses critiques, ce qui explique le rejet du terme de "féminisme", considéré comme une forme de radicalité, une "idéologie" souhaitant voir disparaittre la femme même. L'analyse que fait Antoinette Fouque du slogan "Notre corps nous appartient", met en lumière toute la divergence des courants du MLF:

"Notre corps nous appartient pouvait conduire d'un côté à l'avortement et au genre, à la dégénitalisation, à la désexuation, ce qui était la direction de Monique Wittig, avec le désir affirmé de l'abolition du terme "femme" et de ce qu'aujourd'hui certaine appelle "l'insupportable maternité". Les deux orientations féministes, celle du Queer et celle du féminisme radical d'extrême gauche, programment la disparition des femmes. De l'autre au désir d'enfant - que je cherchais même au cœur de l'avortement ou dans les ratages de la stérilité - à la question de la corporéité profonde, du corps producteur et de la génésique" (MLF, 2018b, p. 107).

Le courant Psychanalyse et Politique du MLF récuse le terme de féminisme qui évoque, pour ses tenantes, l'idéologie radicale d'une disparition des femmes par l'égalité. Le terme de "gynocide" est inventé par Antoinette Fouque pour marquer cette volonté de disparition. En toute logique, les femmes proches de Psychanalyse et politique condamnent le Queer et le genre comme les avatars du "gynocide". Pour elles, à l'instar d'Hélène Cixous en grande confrontation avec Monique Wittig, il s'agit de "déconstruire le féminisme comme idéologie et [de] faire émerger un sujet femme" (Cixous, 1977, citée par Lassere, 2018, en ligne). En 1977, dans le dossier "Écriture, féminité, féminisme", coordonné par Françoise van Rossum-Guyon et publié dans la Revue des sciences humaines, Hélène Cixous refuse de se dire féministe pour se déclarer, dans un même élan, résolument antiféministe: "Pour moi, de façon extrêmement précise", explique-t-elle, "le féminisme aujourd'hui est une idéologie, qui à la limite, est réactionnaire”. Elle ajoute: “[l]e féminisme n'est pas la lutte des femmes, la lutte des femmes passe par une lutte contre le féminisme. Le féminisme, comme idéologie (de l'avant-garde bourgeoise au réformisme), maintient le pouvoir en place dans un processus répétitif, oppositionnel, provocatoire" (Cixous, 1977, citée par Lassere, 2018, en ligne).

Ainsi, pour les femmes du courant Psychanalyse et politique, le féminisme reconduit l'ordre masculin et l'idéologie de domination. Seule vaut la différence des sexes. Paradoxalement, ce courant différentialiste, qui rejette le terme même de féminisme sera reçu, aux États-Unis, sous le nom de French Feminism, avec pour figure de proue les psychanalystes Luce Irigaray et Julia Kristeva et l'écrivaine Hélène Cixous. Ainsi, en France comme outre- Atlantique à cette époque, la psychanalyse est perçue comme étant résolument différentialiste. Ce French Feminism serait donc un antiféminisme....

Selon PsychéPo, on peut certes accorder, à l'intérieur de la psychanalyse, des “révisions", mais la différence des sexes est le combat politique principal : l'égalité et l'égalitarisme révolutionnaire sont considérés comme des idées réactionnaires, qui ont pour effet de faire disparaître les sexes. Pour Antoinette Fouque, il s'agit 
de viser la parité et d'affirmer le féminin, la femme, "l'hétérosexuation de la société". "Comment parler d'hétérosexualité s'il n'y a qu'un seul sexe?”, dit-elle encore dans un bon nombre de textes ou de conférences.

C'est dans ce contexte mouvementé de débats autour des questions de sexualité féminine et de féminisme que Lacan donne le séminaire Encore, en 1972-1973 (notons que Antoinette Fouque étant son analysante à l'époque). Lors de la séance du 13 mars 1973, il théorise les formules de la sexuation ainsi que les notions de jouissance féminine, de jouissance phallique, de jouissance Autre. Dans ce même séminaire, il appelle, pour la psychanalyse, à “un renouvellement dans le domaine de l'Éros” (Lacan, 1975, p. 63).

Dans leurs réflexions théoriques, les psychanalystes différentialistes tentent d'opérer un déplacement au sein même du corpus théorique analytique : elles mettent en perspective des critiques du texte freudien et lacanien et travaillent autour de la déconstruction de la libido unique afin de promouvoir la "libido 2" et la "libido creandi", chères à Antoinette Fouque. Devant ces tentatives d'innovation, du moins de relecture du corpus freudien, l'accueil de Lacan est plutôt froid et radical: alors que Antoinette Fouque et Serge Leclaire, dont elle est proche, souhaitent organiser un séminaire sur le "Deux" à l'École freudienne de Paris (l'EFP, fondée et dirigée par Lacan), il fait envoyer le 15 septembre 1977 ce mot à Serge Leclaire: “Bien cher, il n'est pas question que vous fassiez le séminaire dont Simatos m'a communiqué l'annonce à l'École freudienne de Paris" (MLF, 2018b, p. 246). Le "renouvellement dans le domaine de l'Éros" ne passera pas par là...

À partir des années 1980-1990, le courant Psychanalyse et Politique du MLF, essentiellement concentré sur les publications de revues et des Éditions des femmes fondées par Antoinette Fouque, s'essouffle peu à peu. Pour autant le paradoxe demeure : dans la doxa psychanalytique, il n'y a de psychanalyse féministe que différentialiste, et seule vaut la différence des sexes: la promotion de "la" femme et du féminin est la condition de l'émancipation, et celle-ci passe essentiellement par la procréation. En 1979, le MLF devient une marque déposée, liée définitivement au mouvement d'Antoinette Fouque. La rupture avec l'autre courant du MLF est définitivement consommée.

Il apparaît ainsi que ce n'est pas au sein de ce courant différentialiste et naturalisant le féminin que peuvent se développer les réflexions autour du genre, des questions transgenres ou encore du système hétéronormatif. Les conséquences politiques sont visibles:

- on constate un rejet parfois poli, parfois virulent de tout ce qui vient déconstruire les systèmes d'hétéronormativité, les normes de genre,

- une transphobie parfois violente s'exprime dans la doxa psychanalytique,

- des réactions virulentes concernant la PMA (procréation médicalement assistée), la GPA (gestation pour autrui) et le mariage pour tous s'élèvent également.

En France, en 2013, dans le contexte de la loi Taubira sur le mariage homosexuel dit "pour tous", un certain nombre de psychanalystes différentialistes (comme Michèle Montrelay proche d'Antoinette Fouque et d'autres encore) se sont opposé à la modification de la loi sur le mariage, arguant du fait que "pour l'enfant il faut les deux. Les mots père et mère désignent cette différence. Ils préservent sa nécessité. Avec le mariage pour tous, la différence s'efface" (Montrelay, 2013, en ligne).

Remarquons également que le terme même de "genre" est mal reçu chez la plupart des psychanalystes. Il existe bien sûr des exceptions, comme, en France, Jean Allouch, son école et les éditions EPEL, 9 ou Monique David Ménard, Michel Tort, et chez les plus jeunes, Thamy Ayouch, Fabrice Bourlez ou encore Beatriz Santos et Vincent Bourseul, entre autres.

Si, à l'intérieur de la théorie psychanalytique, il apparaît manifeste que l'articulation au politique bute contre la difficulté à dépasser la question de la différence des sexes, cette impasse semble reconduire les discours essentialisants sur les femmes ou le féminin procréatif. L'autre courant du MLF mené par Monique Wittig et Christine Delphy s'est résolument placé à l'extérieur de la psychanalyse et contre son discours. Ainsi, Monique Wittig promeut le terme de féminisme dans son acception radicale:

"L’ambiguïté du terme "féministe" résume toute la situation. Que veut dire "féministe" ? Féministe est formé avec le mot "femme" et veut dire "quelqu'un qui lutte pour les femmes". Pour beaucoup d'entre nous, cela veut dire "quelqu'un qui lutte 
pour les femmes en tant que classe et pour la disparition de cette classe". Pour de nombreuses autres, cela veut dire "quelqu'un qui lutte pour la femme et pour sa défense" - pour le mythe donc et son renforcement" (Wittig, 2013 [2001], p. 50).

Démythifier "le continent noir", déconstruire la supposée "énigme de la féminité" et les discours sur "la jouissance Autre » passent par une position de rejet radical du discours et de la théorie psychanalytiques. Christine Delphy fait ce constat:

"La psychanalyse et la sociologie ne prennent pas en compte l'oppression des femmes. Ne la prenant pas en compte, elles la reprennent à leur compte: elles l'intègrent comme un donné. Elles étudient des domaines de la vie sociale et de l'expérience subjective où et par lesquels les femmes sont opprimées sans que cette oppression apparaisse comme telle" (Delphy, 2001 [1998], p. 263).

Les rapports de domination, de pouvoir, d'oppression sont, pour les féministes matérialistes, reconduits par l'énonciation et le discours psychanalytiques. Si, pour Foucault, la psychanalyse participe d'un dispositif disciplinaire relevant de la biopolitique et de la gouvernementalité des corps, pour Wittig, elle fait partie de la pensée dominante, de la pensée straight, en raison de son incapacité à réfléchir sur l'émergence de son propre discours. Cette pensée dominante, selon elle, opprime les femmes et les place dans une mythologie indépassable.

"Quelle est donc cette pensée qui refuse de faire retour sur elle-même, qui ne remet jamais en cause ce qui la constitue au premier chef ? Cette pensée est la pensée dominante. Cette pensée affirme qu'il existe un déjà-là des sexes, quelque chose qui précède toute pensée, précède toute société. Cette pensée est la pensée de ceux qui gouvernent les femmes" (Wittig, 2013 [2001], p. 44).

Elle ajoute :

"Les discours qui nous oppriment tout particulièrement nous lesbiennes féministes et hommes homosexuels et qui prennent pour acquis que ce qui fonde la société, toute société, c'est l'hétérosexualité, ces discours nous nient toute possibilité de créer nos propres catégories, ils nous empêchent de parler sinon dans leurs termes et tout ce qui les remet en question est aussitôt méconnu comme "primaire". Notre refus de l'interprétation totalisante de la psychanalyse fait dire que nous négligeons la dimension symbolique. Ces discours parlent de nous et prétendent dirent la vérité sur nous dans un champ apolitique" (Wittig, 2013 [2001], p. 60).

Les critiques de Wittig dénoncent la naturalisation et l'essentialisation de la différence des sexes, raison pour laquelle elle rejette les théorisations d'Antoinette Fouque sur la procréation. Monique Wittig met au jour également l'anhistoricisation des notions psychanalytiques, l'universalisme interprétatif et l'invariant de ses concepts, qui entrainent une forme d'apolitisme de la psychanalyse et une dépolitisation de son écoute:

"La pensée Straight se livre à une interprétation totalisante à la fois de l'histoire, de la réalité sociale, de la culture et des sociétés, du langage et de tous les phénomènes subjectifs. Je ne peux que souligner ici le caractère oppressif que revêt la pensée Straight dans sa tendance à immédiatement universaliser sa production de concept, à former des lois générales qui valent pour toutes les sociétés, toutes les époques, tous les individus. C'est ainsi qu'on parle de l'échange des femmes, la différence des sexes, l'ordre symbolique, $l$ 'inconscient, le désir, la jouissance, la culture, l'histoire" (Wittig, 2013 [2001], p. 63).

Dans cette perspective, "le concept de différence des sexes” relève pour Wittig d'un énoncé, d'un discours politique, qui vise à renforcer la domination d'une classe sur une autre : "C'est bien dire que pour nous il ne peut y avoir de femmes, ni d'hommes, qu'en tant que classe et qu'en tant que catégories de pensée et de langage, ils doivent disparaître politiquement, économiquement, idéologiquement" (2013 [2001], p. 64). La radicalité de Wittig l'amène à penser qu'il faut dépasser le terme de féminisme et de femme pour marquer la lutte politique: le lesbianisme politique est l'issue révolutionnaire. Face à la "féminologie" et au "il y a deux sexes" d'Antoinette Fouque, Wittig oppose cette affirmation devenue célèbre: "Les lesbiennes ne sont pas des femmes". Cette assertion a eu des conséquences politiques en France au sein du courant des féministes matérialistes, menant paradoxalement à des positions proches du courant différentialiste. Ainsi, par exemple, 
des voix se sont élevées pour dénoncer la dimension hétéronormative du mariage, et de ce fait pour s'opposer à une loi promouvant le "mariage pour tous".

La radicalisation des positions a produit ainsi une extinction de la fécondité des débats et une multiplication des injonctions normatives. La spontanéité du mouvement, le désordre conceptuel et la liberté de penser ont laissé place à l'obligation de choisir son camp, et à se positionner du "bon côté" de la lutte.

Dans une certaine mesure, et de façon évidemment paradoxale, l'hétérosexuation sociale de Fouque et le lesbianisme politique de Wittig ont promu une démarche épistémologique similaire aboutissant à de nouvelles catégories discursives et normatives. Ce qui avait fait la spontanéité joyeuse du commencement du mouvement des femmes, ses gestes de renversement inédits et innovants, a été peu à peu rattrapé par le sérieux et les passions tristes des enjeux de pouvoir et des enjeux théoriques. Et la psychanalyse en a perdu sa libido!

\section{5. "LA PSYCHANALYSE EST UNE THÉORIE DU GENRE"}

Pourtant, malgré les dissensions, les ruptures et les rejets, ce que la fécondité intellectuelle et théorique des années 1970 a apporté à la psychanalyse est essentiel: désormais, la théorie psychanalytique ne peut plus penser la sexualité en dehors du politique et du discours social et normatif, en dehors des dispositifs disciplinaires et de la biopolitique des corps, en dehors d'une "généalogie de la psychanalyse", telle que la met en perspective Foucault dans son Histoire de la sexualité (1976). Qu'il soit différentialiste et hétérosexualiste, qu'il soit matérialiste et lesbianiste, le mouvement des femmes n'a pas seulement modifié les discours normatifs et les pratiques sociales inégalitaires, il a permis un déplacement, un certain renversement de la question du corps. Le corps devient un récit politique. "Le corps n'est pas une chose mais une situation", écrivait Simone De Beauvoir (1949, p. 75). Ces débats et ces luttes ont ainsi permis une politisation radicale de la notion de différence des sexes, de sexe et de sexualité. On a pu même commencer à penser, avec Wittig, à une société sans sexe. Celle-ci met en perspective l'abandon du dualisme sexe/genre: "En 1949, Simone de Beauvoir détruisait le mythe de la femme. Il y a dix ans, nous nous mettions debout pour nous battre pour une société sans sexe" (2013 [2001], p. 50). Il s'agit donc de "montrer comment 'le sexe' est sous la dépendance historique de la sexualité". C'est ce propose Foucault dans La Volonté de savoir:

\footnotetext{
"Ne pas placer le sexe du côté du réel, et la sexualité du côté des idées confuses et des illusions ; la sexualité est une figure historique très réelle, et c'est elle qui a suscité comme élément spéculatif, nécessaire à son fonctionnement, la notion du sexe. Ne pas croire qu'en disant oui au sexe, on dit non au pouvoir ; on suit au contraire le fil du dispositif général de sexualité. C'est de l'instance du sexe qu'il faut s'affranchir si, par un retournement tactique des divers mécanismes de la sexualité, on veut faire valoir contre les prises du pouvoir, les corps, les plaisirs, les savoirs, dans leur multiplicité et leur possibilité de résistance. Contre le dispositif de sexualité, le point d'appui de la contre-attaque ne doit pas être le sexe-désir, mais les corps et les plaisirs" (Foucault, 1976, p. 208).
}

Une psychanalyse sans sexualité est-elle possible? Peut-on envisager de défaire le sexe de la sexualité et de travailler au "renouvellement dans le domaine de l'Éros", selon le vœu de Lacan? Une psychanalyse féministe serait-elle une psychanalyse délestée de la sexualité? Délestée du sexe-désir pour ouvrir au corps-plaisir ? Une psychanalyse disposée à la joie ?

En 1975, aux États-Unis, au cœur des débats féministes, l'anthropologue Gayle Rubin faisait le constat suivant: "La psychanalyse est un féminisme manqué" (2010a [1975], p. 54). Selon Gayle Rubin, une certaine psychanalyse rate ce "retournement tactique". Elle refuse de procéder à une resignification de certaines positions théoriques, à une critique épistémologique de ces concepts, instituant ainsi des dogmes qui se fossilisent. Gayle Rubin, dans son essai “Le marché aux femmes” fait cette analyse précieuse:

"La bataille entre la psychanalyse et le mouvement des femmes et le mouvement gay est devenue légendaire. Cette confrontation entre les révolutionnaires sexuels et l'establishment des cliniciens a été provoquée en partie par l'évolution de la psychanalyse aux États-Unis, où la tradition clinique a fétichisé l'anatomie. [...] Transformant la loi morale en loi scientifique, la pratique clinique a servi à imposer la norme sexuelle à des participants indisciplinés. En ce sens, la psychanalyse 
est souvent devenue plus qu'une théorie des mécanismes de reproduction des arrangements sexuels; elle a été l'un de ces mécanismes. Le but des révoltes féministes et gay étant de démanteler le dispositif qui enrégimente la sexualité, une critique de la psychanalyse était de mise. [...] Les effets que des systèmes sociaux dominés par les hommes produisent sur les femmes ne sont nulle part mieux attestés que dans la littérature clinique. Selon l'orthodoxie freudienne, la conquête de la féminité "normale" est extorquée aux femmes au prix fort. La théorie de l'acquisition du genre aurait pu être le point de départ d'une critique des rôles de sexe. Au lieu de cela, les implications radicales de la théorie ont été radicalement refoulées [...]. De plus, s'il faut sauver la psychanalyse de ses propres motivations de refoulement, ce n'est pas par égard pour la réputation de Freud. La psychanalyse présente un ensemble unique de concepts permettant de comprendre les hommes, les femmes et la sexualité. C'est une théorie de la sexualité dans la société humaine. Et le plus important est que la psychanalyse fournit une description des mécanismes par lesquels les sexes sont divisés et déformés, une description de la manière dont des petits enfants bisexuels et androgynes sont transformés en garçons et en filles. La psychanalyse est une théorie féministe manquée" (Rubin, 2010a [1975], p. 53-54).

Elle ajoute, un peu plus loin :

"Une révolution féministe complète libèrerait plus que les femmes. Elle libèrerait les formes d'expressions sexuelle, et elle libèrerait la personnalité humaine de la camisole de force du genre. Comme la psychanalyse est une théorie du genre, l'écarter serait suicidaire pour un mouvement politique qui se consacre à éradiquer la hiérarchie de genre (ou le genre lui-même) ” (p. 68)..$^{10}$

Ces deux assertions sur la psychanalyse - "la psychanalyse est une théorie du genre" et "la psychanalyse est un féminisme manqué" - marquent une importante rupture avec les définitions véhiculées par ce que l'on peut considérer, ironiquement, comme l'Industrie Psychanalytique Actuelle (acronyme IPA...): celle-ci promeut la massification des diagnostics psychopathologiques et des modes d'emploi psychothérapeutiques, s'enferme dans l'obsession de l'étiologie sexuelle ou le volontarisme psychogénétique, ${ }^{11}$ prétend à l'anhistoricité de la sexualité et alimente ses excès de signification, imagine l'essentialisme et la naturalisation de tout type de différences, pourvu que celle-ci prenne racine dans celle, paradigmatique, reine de toutes les différences: celle des sexes.

Contre un conformisme sexuel porté par un moralisme sexuel, il s'agit pour Gayle Rubin de "créer une éthique sexuelle pluraliste” (2010b [1984], p. 163), dans le cadre d'un projet théorique et politique et dans la perspective foucaldienne des corps et des plaisirs. Dans cet essai, elle met en relief les différences corporelles qui relèvent de l'expérience et de ses pratiques. Malgré les mises en ordre sociales qui tentent de catégoriser et de pathologiser la sexualité et les pratiques du corps, celles-ci, par l'expérience des plaisirs divers qui les rendent indéfinissables, deviennent un lieu de résistance politique. ${ }^{12}$ Serait-ce donc de l'extérieur de la théorie psychanalytique, notamment avec Gayle Rubin et Judith Butler, qu'un renouvellement de sa théorie semblerait possible?

Si Lacan a toujours prôné un "retour à Freud", Judith Butler ajoute en effet qu'elle souhaite "opérer un retour critique à Freud” (2009 [1993], p. 69), notamment dans la lecture qu'elle fait de l'article de Freud "Pour introduire le narcissisme" [1914] et de celui de Lacan, "Le stade du miroir » [1936/1949], avec un opérateur conceptuel qu'elle nomme "le phallus lesbien (Lesbian's phallus)". Elle écrit: "Il pourrait sembler que le phallus lesbien n'a pas grand-chose à voir avec cela, mais je vous assure (je vous promets ?) que je n' aurais pas pu ne pas passer par là" (Butler, 2009 [1993], p. 69). Sans entrer dans le détail théorique de cette lecture métapsychologique ${ }^{13}$ des zones érogènes, de la libido et du narcissisme, ce qui m’intéresse ici, c'est l'usage que fait Butler des termes issus du corpus théorique analytique: qualifier le "phallus" de "lesbien", ne seraitce pas tenter, ironiquement(?), de faire dialoguer à nouveau Fouque et Wittig autour d'une bière ou d'une tasse de thé conceptuelles? 


\section{LE RIRE DES FÉMINISTES}

Les catégories ontologiques (“femme”, "homme”, "homosexuel”, "hétérosexuel”, “noir”, "blanc”, etc.) essentialisent et naturalisent des normes de genre, des normes sexuelles ou des normes identitaires. Les productions conceptuelles et théoriques lorsqu'elles ne prennent pas en compte leurs conditions historiques de production risquent de se transformer en dogmes fossilisés.

Or, l'expérience analytique insiste sur l'idée que toute construction identitaire peut devenir une entrave pour le sujet, une sorte d'idéalisation qui peut confiner à une forme de mélancolisation. Les normes de genre, les cliché sexuels, "l'idéal de la femme blanche" décrit par Despentes, l'homme jeune blanc, actif, cisgenre et hétérosexuel, c'est-à-dire les supposés représentants d'une santé universelle, sont déconstruits par l'expérience de l'analyse. Lors d'une analyse, on peut pleurer sur soi, mais on peut rire de soi aussi et sourire des discours normatifs dont on peut être dupe.

À l'occasion de son séminaire sur la théorie du moi, Lacan avance que:

"Le moi est la somme des identifications du sujet, avec tout ce que cela peut comporter de radicalement contingent. Si vous me permettez de l'imager, le moi est comme la superposition des différents manteaux empruntés à ce que j'appellerai le bricà-brac de son magasin d'accessoires" (1980 [1955-1956], p. 187).

Contingence et bricolage d'identifications voici donc ce qu'est le moi. L'expérience d'une analyse revient aussi à se rendre compte de cette contingence et de se défaire des fictions que l'on construit sur soi-même et des effets pathologiques et entravants qu'elles peuvent avoir. Comme l'écrit Pedro Ambra, lorsqu'il analyse ladite "prolifération identitaire » des mouvements LGBTAIQQ+, "force est de constater que - loin de constituer des défenses phalliques contre le réel de la différence sexuelle - cette inscription vise justement l'impossible du sexuel en instituant un horizon de contingence, étant donné que de nouvelles lettres sont toujours prêtes à surgir et à se réarranger, dans un mouvement échappant aux lois symboliques et aux fixations imaginaires"(Ambra, 2018, p. 25). Cette contingence déconstruit le sérieux des catégories. C'est pourquoi Judith Butler écrit : "face à des catégories sérieuses, le rire est indispensable pour le féminisme" (2005 [1990], p. 52). Qu'est-ce qu'en effet, une psychanalyse qui pense en catégorie ? Lors de la séance du 5 mai 1965 du séminaire Problèmes cruciaux pour la psychanalyse, Lacan faisait cette analyse :

"Un sujet est psychanalyste, non pas savant rempardé derrière des catégories au milieu desquelles il essaie de se débrouiller pour faire des tiroirs dans lesquels il aura à ranger les symptômes qu'il enregistre, de son patient, psychotique, névrotique ou autre, mais pour autant qu'il entre dans le jeu signifiant" (Manuscrit inédit en ligne).

Le sexuel délesté de la sexualité se transforme en une force érotique, en passion joyeuse lorsqu'il est contingence, jeux, possibles, bricolage.

Rappelons la liste spinoziste des "passions tristes" que Deleuze analyse dans son ouvrage sur le philosophe: d'abord la tristesse elle-même, puis la haine, y compris la haine retournée contre soi, l'aversion, la moquerie, la crainte, le désespoir, la pitié, l'indignation, l'envie, l'humilité, le repentir, l'abjection, la honte, le regret, la colère, la vengeance, la cruauté, tout ce qui fait naître un sentiment d'esclavage, de soumission. "C'est aux esclaves, non aux hommes libres, qu'on donne des récompenses pour leur bonne conduite” (Deleuze, 1999 [1981], p. 37-42). L'esclave, le tyran, le prêtre sont des catégories sérieuses. Ce qui les unit, analyse Deleuze dans Spinoza philosophie pratique, c'est la haine de la vie, le ressentiment contre la vie (1999). L'esclave, c'est l'homme aux passions tristes; le tyran, c'est l'homme qui exploite les passions tristes; le prêtre, c'est l'homme qui s'attriste sur la condition humaine et les passions de l'homme en général. Les féministes ont compris que le tyran a besoin de la tristesse des autres pour réussir à prendre le pouvoir, tout comme les autres ont besoin de tyran pour déverser leur tristesse. Affecté par les passions tristes, le corps est empêché, diminué dans sa puissance d'agir. La joie, elle, donne une puissance d'agir augmentée, élargie. Alors, comment créer de la joie dans le mouvement? 
Dans le recueil 40 slogans féministes, Béatrice Fraenkel (App, Faure-Fraisse, Fraenkel et Rauzier, 2010) rappelle le contexte et l'atmosphère des événements des années 1970 et 1980 : "Slogans, banderoles, tracts, chansons, actions et tous les éléments constitutifs des rassemblements ont été réinventés dès les débuts du MLF. La manifestation féministe innove, elle est un événement au sein de la longue histoire des manifestations militantes" (App et alii., 2010,p. 5). “C'est en slogan qu'on devient féministe”, tel est le slogan des slogans qu'on entend dans une foule de féministes. En slogan, c'est-à-dire en prenant la rue et la parole: beau zeugma pour réunir deux espaces qui ont été longtemps interdits et rendus impossibles aux femmes.

"Les féministes aiment les jeux de langage" (App et alii., 2010, p. 6). Face aux mots d'ordre et aux injonctions, les féministes des années 1970 en France ont produit des mots d'esprit, de la joie, de la liberté de penser. On a pu lire sur les pancartes, on a pu entendre crier :

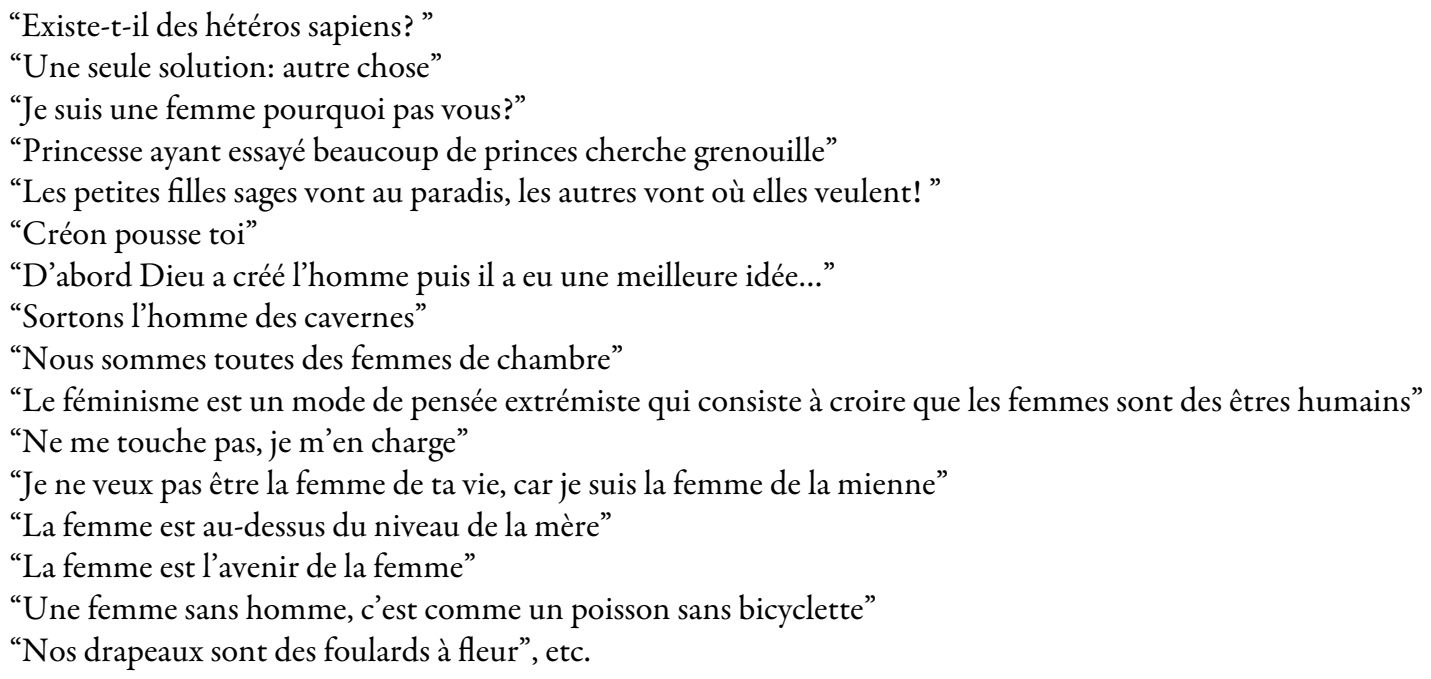

Une psychanalyse féministe permettrait, me semble-t-il, une disposition ouverte aux possibles, aux bricolages, aux jeux, aux rires de soi et de ses propres modes de jouissance, en somme un certain art de la joie.

\section{REFERENCIAS BIBLIOGRÁFICAS}

Allouch, J. (s. d.). Présentation de la collection Les grands classiques de l'érotologie moderne. Récupéré de http://www.e pel-edition.com/collection/9/les-grands-classiques-de-l-n-rotologie-moderne.html

Ambra, P. (2018). Des formules au nom: Bases pour une théorie de la sexuation chez Lacan (Thèse doctorale inédite, sous la direction de Nelson Jr Da Silva et Laurie Laufer). Université de Sāo Paulo: Brasil.

App, C., Faure-Fraisse, A.-M., Fraenkel, B. et Rauzier, L. (2010). 40 ans de slogans féministes 1970-2010. DonnemarieDontilly: Éditions iXe.

Bard, C. et Chaperon, S. (2017). Le dictionnaire des féministes, France XVIIIe-XXIe siècle. Paris: Presses Universitaires de France.

Butler, J. (2005 [1990]). Trouble dans le genre (Trad. C. Kraus). Paris: La Découverte.

Butler, J. (2009 [1993]). Ces corps qui comptent, De la matérialité et des limites discursives du "sexe" (Trad. C. Nordmann). Paris: Éditions Amsterdam.

Cixous, H. (1977). Entretien avec Françoise Van Rossum-Guyon. Revue des sciences humaines dossier "Écriture, féminité, féminisme", 168, 479-493.

Cixous, H. (2010 [1975]). Le rire de la méduse et autres ironies. Paris: Éditions Galilée.

De Beauvoir, S. (1949). Le deuxième sexe. Paris: La Découverte.

Deleuze, G. (1999 [1981]). Spinoza, Philosophie pratique. Paris: Les Éditions de Minuit. 
Delphy, C. (2001 [1998]). L'ennemi principal t. 1 Économie politique du patriarcat, t. 2 Penser le genre (2e ed.). Paris: Syllepse.

Despentes, V. (2006). King Kong Theory. Paris: Grasset.

Dumas fils, A. (1872). L'homme-fermme, Réponse à M. Henri d'Ideville. Paris: Michel Lévy Éditeurs.

Duras, M. et Gauthier, X. (2013 [1974]). Les Parleuses. Paris: Les Éditions de Minuit.

Foucault, M. (1976). Histoire de la sexualité, 1. La volonté de savoir. Paris: Gallimard.

Fouque, A. (2004 [1995]). Il y a deux sexes, essais de féminologie. Paris: Gallimard.

Fraisse, G. (2007). Le signal égal ou la logique dans l'histoire. In S.C. Hause (éd.) Hubertine Auclert Pionnière du féminisme, textes choisis. Saint-Pourçain-sur-Sioule, France: Éditions Bleu autour.

Freud, S. (1969 [1914]). Pour introduire le narcissisme. In S. Freud, La vie sexuelle (Trad. J. Laplanche.) (pp. 81-105). Paris: Presses Universitaires de France.

Freud, S. (1962 [1985]). La question de l'analyse profane (Trad. J. Altounian, O. Bourguignon, P. Cotet). Paris: Gallimard.

Freud, S. (1990 [1895]). Études sur l'hystérie (Trad. A. Berman). Paris: Presses Universitaires de France.

Freud, S. (1992 [1920]). Sur la psychogénèse d'un cas d'homosexualité féminine. In S. Freud, Névrose, psychose, perversion (pp. 245-270) (Trad. D. Guérineau). Paris: Presses Universitaires de France.

Hause, S. C. (éd.) (2007). Hubertine Auclert. Pionnière du féminisme, textes choisis. Saint-Pourçain-sur-Sioule, France: Éditions Bleu autour.

Hause, S. C. (2007). Présentation. In S.C. Hause (éd.), Hubertine Auclert. Pionnière du féminisme, textes choisis (pp. 19-63). Saint-Pourçain-sur-Sioule, France : Éditions Bleu autour.

Irigaray, L. (1974). Speculum. De l'autre femme. Paris: Les Éditions de Minuit.

Irigaray, L. (1984). Éthique de la différence sexuelle. Paris: Les Éditions de Minuit.

Irigaray, L. (2003). Ce sexe qui n'en est pas un. Paris: Les Éditions de Minuit.

Lacan, J. (1966 [1949]). Le stade du miroir comme formateur de la fonction du Je. In J. Lacan, Écrits (pp. 93-100). Paris: Éditions Seuil.

Lacan, J. (1966 [1953]). Fonction et champ de la parole et du langage en psychanalyse. In J. Lacan, Écrits (p. 237-322). Paris: Éditions Seuil.

Lacan, J. (1980 [1955-1956]). Le Moi dans la théorie de Freud et dans la technique psychanalytique, Le séminaire, II. Paris : Éditions Seuil.

Lacan, J. (1994 [1956-1957]). La relation d'objet, Le séminaire IV. Paris: Éditions Seuil.

Lacan, J. (2004 [1962-1963]). L'Angoisse. Le séminaire X. Paris : Éditions Seuil.

Lacan, J. (1965). Problèmes cruciaux pour la psychanalyse (inédit).

Lacan, J. (1966). Écrits. Paris: Seuil.

Lacan, J. (1975 [1972-1973]). Encore. Le séminaire XX. Paris: Éditions Seuil.

Lassere, A. (2018). Des féministes qui ne sont pas féministes ? Écrivaines et lutte des femmes en France des années 1970 aux années 1980. GLAD ! Rhétoriques antiféministes, 4. Récupéré de https://www.revue-glad.org/1133

Leff, G. (2009). Portraits de femmes en analystes. Lacan et le contre-transfert. Paris: EPEL.

Mitchell, J. (1975 [1974]). Psychanalyse et féminisme, 1. Paris: Éditions Des Femmes.

Mouvement de Libération des Femmes (2018a). Psychanalyse et politique 1968-2018. 50 ans de libération des femmes, Vol I. Les premières années. Paris: Éditions Des Femmes.

Mouvement de Libération des Femmes (2018b). Psychanalyse et politique 1968-2018. 50 ans de libération des femmes, Vol. II. La plus longue des révolutions. Paris: Éditions Des Femmes.

Montrelay, M. (1977). L’Ombre et le nom. Sur la féminité. Paris: Éditions de Minuit. 
Montrelay, M. (23 février 2013). Sur l'égalité des droits et le mariage pour tous [Exclusivement en ligne]. Récupéré de http://mouvement-cout-freudien.fr/propos-de-michele-montrelay-sur-legalite-des-droits-et-le-mariage-pou r-tous $/ 395$

Preciado, B. (2008). Testo Junky. Sexe, drogue et politique. Paris: Grasset.

Rieder, I. et Voigt, D. (2003). Sidonie Csillag, homosexuelle chez Freud, lesbienne dans le siècle (Trad T. Gindele). Paris : EPEL.

Rubin, G. (2010a [1975]). Le marché aux femmes. "Économie politique" du sexe et systèmes de sexe/genre (Trad. N.-C. Mathieu en coll. avec G. Pheterson). In G. Rubin, Surveiller et jouir, anthropologie politique du sexe (pp. 135-224). Paris: EPEL.

Rubin, G. (2010b [1984]). Penser le sexe : Pour une théorie radicale de la politique de la sexualité (Trad. F. Bolter). In G. Rubin, Surveiller et jouir, anthropologie politique du sexe (pp. 135-224). Paris : EPEL.

Santos, B. (30 janvier 2016). Pour une dissémination (du) phallique. Judith Butler et la construction du corps [Exclusivement en ligne]. Récupéré de http://www.pointsdaccroche.com/dossier/pour-une-dissemination-duphallique-judith-butler-et-la-construction-du-corps/

Wittig, M. (1969). Les Guérillères. Paris: Les Éditions de Minuit.

Wittig, M. (2013 [2001]). La pensée straight. Paris: Éditions Amsterdam.

\section{Notas}

1 Il s'agit ici d'une référence au texte Les parleuses, dialogue entre Marguerite Duras et Xavière Gauthier (2013 [1974]) présenté comme un "écrit de la parole sans peur du CENSEUR".

2 Le terme allemand Penisneid signifie chez Freud "l'envie du pénis".

3 Je renvoie ici à l'excellent ouvrage de Gloria Leff (2009).

4 Dans sa monographie Dora. Fragment d'une analyse d'hystérie qu'il publie en 1905, Freud rend compte et analyse la cure d'Ida Bauer, jeune fille de 18 ans souffrant de nombreux symptômes hystériques. Il voit dans Ida/Dora le cas paradigmatique de l'hystérie en proie à des désirs oedipiens et à une homosexualité refoulée.

5 En français dans le texte.

6 Entretien sonore diffusé lors de la journée organisée par le CRPMS le 23 novembre 2013 à l'université Paris Diderot, intitulée "Sidonie, la jeune homosexuelle de Freud, une psychanalyse après l'œdipe”.

7 Nous devons la traduction de l'allemand à Jean-Marie Adam et nous l'en remercions.

8 Voir entre autres, Irigaray (1974, 1984, 2003); Montrelay (1977); Cixous (2010 [1975]).

9 Jean Allouch, présentation de la collection Les grands classiques de l'érotologie moderne, EPEL : “Dans les librairies de langue anglaise, les gayand lesbian studies, les travaux de la queer theory ont leurs rayons, leurs revues, leurs collections; ils en sont désormais au stade des compilations. Il n'empêche, persiste en France l'ignorance d'un débat qui a maintenant plus de vingt ans. Conséquence imprévue : ce recul permet aujourd'hui de distinguer les publications et les auteurs qui, au fil du temps, sont devenus des classiques. Est-ce un hasard ? cette émergence, cette réinterrogation d'Éros, ce nouveau rapport à l'érotisme est tout d'abord advenu précisément là où la psychanalyse avait cessé d'être questionnante et inventive. Délaissée par les psychanalystes, la problématisation de l'érotisme contemporain a eu lieu ailleurs qu'au champ freudien. Pas sans liens cependant avec Freud, Lacan, Foucault, Deleuze, Derrida et quelques autres ; ni sans avoir renouvelé la vision de ce qu'était Éros dans le passé. Comme la psychanalyse, ce champ d'études, inédit, insolite, a apporté à certaines disciplines (sociologie, littérature, hellénisme, histoire) des contributions remarquées. Il ne s'agit pas seulement de débats universitaires. Ce sont aussi les rapports de chacun au sexe qui ont été modifiés, en même temps que la place et la fonction du sexe dans la (les) communauté(s). La collection Les grands classiques de l'érotologie moderne vise à ce que s'ouvre un débat critique entre champ gay et lesbien et champ freudien. Le vœu est que soit cerné d'un peu plus près en quoi consistent "les œuvres d'Aphrodite" (Allouch, s. d., en ligne).

10 Je remercie les éditions EPEL de m'avoir autorisée à reproduire ici ce long extrait de Gayle Rubin.

11 Voir ce que dit Lacan dans le séminaire sur les psychoses : "le grand secret de la psychanalyse c'est qu'il n'y a pas de psychogenèse" (1980 [16 novembre 1955], p. 15).

12 Je renvoie ici au livre politique de Beatriz (Paul B.) Preciado (2008).

13 Je renvoie ici à l'excellent article de Beatriz Santos (2016). 